\title{
Increase of Critical Mach Number by Local Linearization Method and Airfoil Construction at Subsonic and Transonic Regimes
}

\author{
Ali. S. Bazgir ${ }^{1}$, Sergey. A. Takovitskii ${ }^{2}$ \\ ${ }^{1}$ Moscow Institute of Physics and Technology, Department of Aeromechanics and Flight Engineering140180, Gagarina Street, 16, \\ Zhukovsky, Russia. \\ ${ }^{2}$ Central Aerohydrodynamic Institute (TsAGI), Zhukovsky Str., Zhukovsky, 140180, Moscow Region, Russia.
}

\begin{abstract}
A symmetrical airfoil has been constructed by local linearization method. A single-point objective function is defined to check the convergence of the method. As an example, the nose and tail zone of supercritical airfoil is fixed and a flat line is placed between them. The optimizable element of the airfoil contour was conjoined with the nose and tail elements of fixed shape at the sections with coordinates $x_{s 1}=0.11$ and $x_{s 2}=0.66$, respectively. The optimizable part of airfoil (the fixed chord line) is divided into $\mathrm{N}=55$ segments. The convergence of this method has been shown with the airfoil constructed with higher critical Mach number rather than the initial airfoil. Finally, this airfoil has been compared with the supercritical airfoil NASA SC (2)-0012 at $M_{\infty}=0.76$. At the second part, several airfoils have been constructed and simulated over different Subsonic and Transonic Mach numbers. Finally, the drag coefficient on constructed airfoils have been compared with supercritical airfoil.
\end{abstract}

\section{Introduction}

Unlike supersonic flows in the range of subsonic velocities, there are no local models connecting the pressure on the body surface with the local geometrical parameters and the conditions of the incoming flow. However, successful examples of applying local linearization in small shape variations based on connecting the pressure and contour curvature $[1,2]$ are known. The variation method, which uses the relationship between variations of pressure and an external normal vector of the optimizable element, was implemented in Ref. [3].

For small deformation of the shape the local pressure coefficient change was estimated by the relation with the element length and the variation of the second derivative of the ordinate y" at subsonic Mach numbers [4]. The same can be estimated for supersonic Mach numbers by the relation of the local pressure coefficient with the element length and the variation of the first derivative of the ordinate $y^{\prime}[5]$.

\section{Local linearization method}

Use Maximum Critical Mach number, $M_{c r}$, achieves when pressure coefficient on the optimizable part of the airfoil has a constant value ( $C_{p}=$ constant $)$. In this case, we have to put $\mathrm{M}=1$ on the optimizable part of the airfoil and get a desired value of pressure coefficient $\left(C_{p_{\text {desired }}}\right)$ by:

$$
C_{p}=\frac{2\left(\frac{P}{P_{\infty}}-1\right)}{\gamma M_{\infty}^{2}}
$$

The optimizable part of the airfoil is divided to several segments and the Mach number is averaged on these elements based on the data of the numerical flow field modeling:

$$
M_{n}=\left[\frac{2}{\gamma-1} \times\left(\frac{\left(1+\frac{\gamma-1}{2} \times M_{\infty}^{2}\right)}{\left(\frac{\gamma}{2} \times M_{\infty}^{2} \times C_{p_{n}}+1\right)}-1\right)\right]^{0.5}
$$

The second order derivative is determined by finite difference approximation through the ordinate values of the three nodal points. A similar approach was implemented for supersonic flows when the linear dependency of the change in pressure on the inclination angle of the element, which is specified by the ordinates of two adjacent points, was used [5]:

$$
\begin{aligned}
& d_{y_{n}}=\frac{\pi}{8} \times\left(x_{n}-x_{n-2}\right) \times\left|1-M_{n-1}^{2}\right|^{0.5} \times\left(C p_{\text {desired }}-\right. \\
& \left.C p_{n-1}\right)+2 \times d_{y_{n-1}}-d_{y_{n-2}} \\
& d_{y_{n}}=\frac{\left(x_{n}-x_{n-2}\right)}{2} \times\left|1-M_{n-1}^{2}\right|^{0.5} \times\left(C p_{\text {desired }}-\right. \\
& \left.C p_{n-1}\right)+d y_{n-1} \text { for } M>1
\end{aligned}
$$

We will use these formulas to connect the changes in the gas-dynamic functions and geometrical parameters in the local analysis. To get new vertical position of each nodal, the following formula is used:

$$
y_{\text {new }}=y_{n}+d_{y_{n}} k
$$

Where $y_{n}$ and $y_{n e w_{n}}$ are the initial airfoil vertical coordinates and new vertical coordinates after applying the linearization of flow parameters $d_{y_{n}}$ at each local segment. 
$k$ is an additional constant value which can be chosen different numbers during the optimization process.

The proposed linear dependence of the surface pressure on the geometrical parameters leads to the quadratic approximation of the objective function and determines the shape variations, which are directed to its reduction:

$$
O F=\left[\frac{\sum_{i}^{n}\left(C p_{i}-C p\right)^{2} l_{i}}{\sum l_{i}}\right]^{0.5}
$$

where $l$ is the length of the contour element, and the summation is done along all elements of the convex part of the contour. $C p$ is desired value of the mean pressure coefficient.

The described model gives a sufficiently rough evaluation of the aerodynamic load change. However, at every iterative step the proposed shape variations are checked within the framework of the numerical computation. In addition, as long as the optimization procedure converges, the variations will decrease and the accuracy of the approximation will increase. In the presented investigation, numerical simulation of the flow over constructed airfoil is performed based on solving Naiver-Stokes equations.

\subsection{Critical Mach number increase}

The nose and tail zone of supercritical airfoil NASA SC (2)-0012 is fixed and a flat line is placed between them. The optimizable part of the airfoil contour was conjoined with the nose and tail elements of fixed shape at the sections with coordinates $x_{s 1}=0.11$ and $x_{s 2}=0.66$, respectively. The optimizable part of airfoil (the fixed chord line) is divided into $\mathrm{N}=55$ segments. The nose element of the contour was specified by $\mathrm{N}=13$ segments and the tail element was specified by $\mathrm{N}=35$ segments. At Mach condition of $M_{\infty}=0.76$, Reynolds number based on reference wind speed and airfoil length is approximately $1.1 \times 10^{6}$. The calculated value of desired pressure coefficient is $C_{p_{\text {desired }}}=-0.5576$.

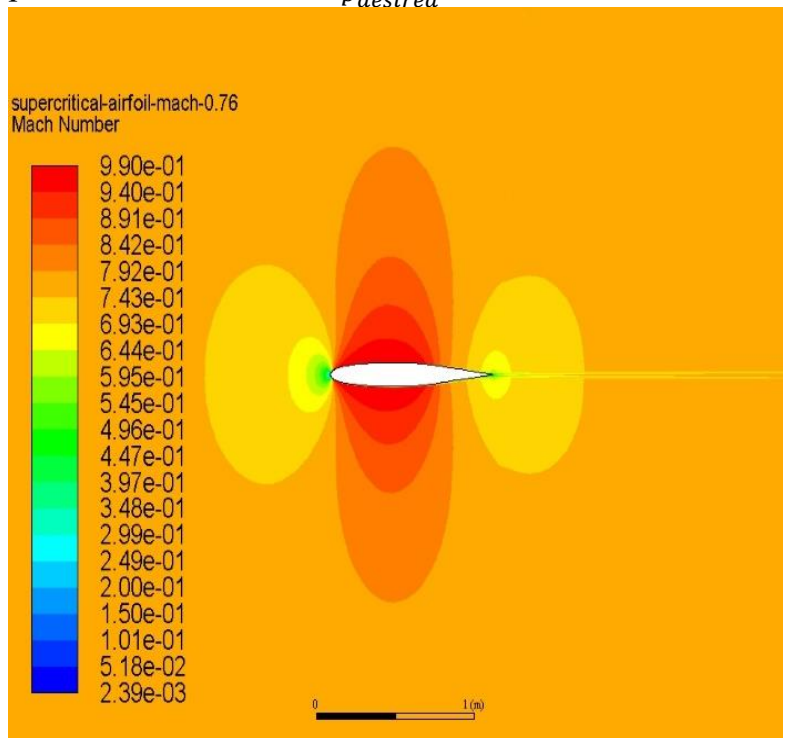

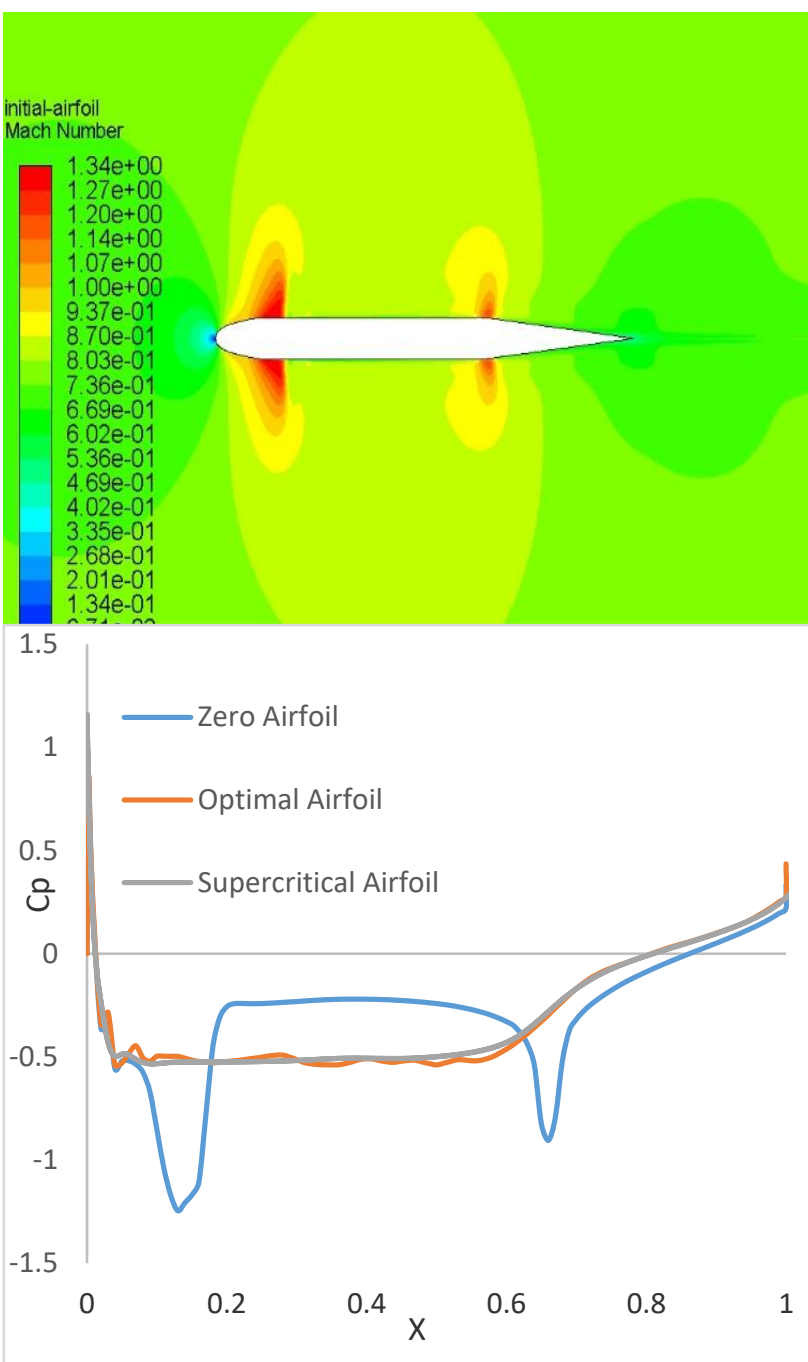

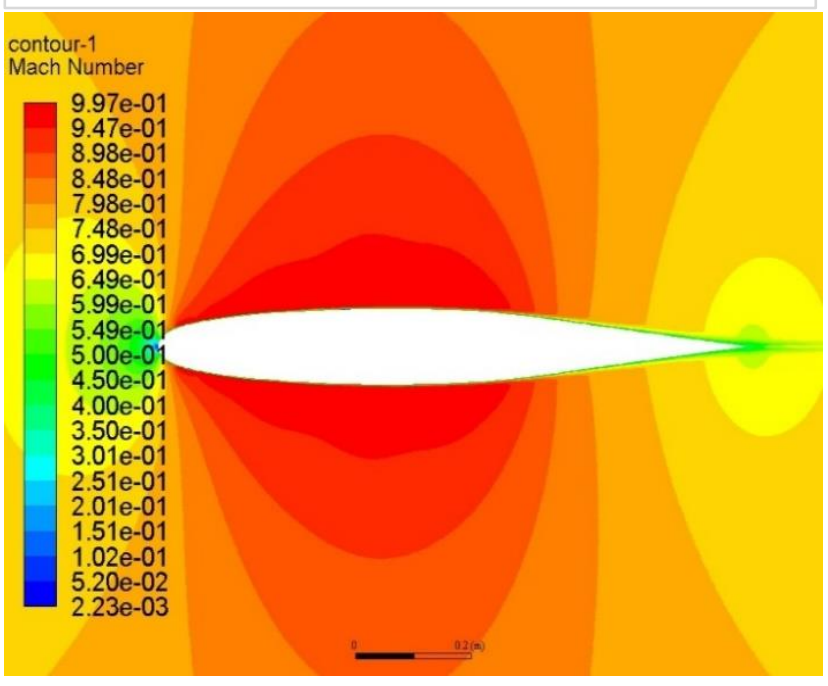

Figure 1. Comparison of the results of initial and optimized airfoil by local linearization.

A The presented figures (fig. 1) compare the pressure coefficient distribution on the optimal airfoil from local linearization, initial airfoil and supercritical airfoil. There are two huge pressure drop in the fixed edges of the initial airfoil where the minimum pressure coefficient reaches to approximately -1.24 . It is because the local velocity of the flow increases hugely and the maximum local Mach 
number on the airfoil's surface reaches to 1.34 in these zones. However, the final optimal airfoil by local linearization method has approximately smooth distribution like the supercritical airfoil. While the shock waves and pressure jumps disappear on the airfoil surface, the overall drag on the airfoil reduces and so Critical Mach number increases.

The optimal airfoil from local linearization method achieves after five cycle of optimization. The deviation of the pressure coefficient value from the desired constant pressure coefficient on the optimizable part of the airfoil is minimum. Maximum local Mach number on the optimal airfoil surfaces reaches approximately to $M=0.997$. In fact, due to the constant pressure distribution on the optimizable part of the airfoil, the value of the Critical Mach number is delayed and there is no local shock waves on the airfoil surface at $M_{\infty}=0.76$. Results from Fig. 2 demonstrates the accuracy of this method in which it shows the convergence of the objective function after few cycles of optimization.

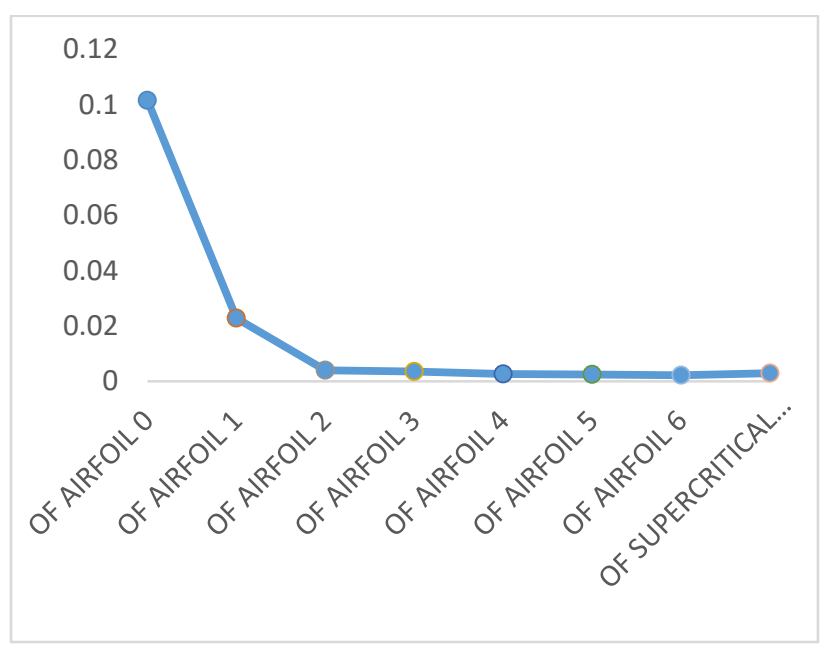

Figure 2. Minimization of objective value during the optimization process.

\section{Drag force mitigation for the Mach number regimes more than critical Mach number by several methods}

The tail and nose of the supercritical airfoil fixed in two points and for the central part of the airfoil (fig. 3), three methods are used to create new airfoil. The first method is to use local linearization method for construction of the central part. The second method is to use an elliptic function to create an elliptical line and then fixe the elliptic line with the nose and tail of the airfoil. Third method is consisted from two inclined flat segments at the central part.

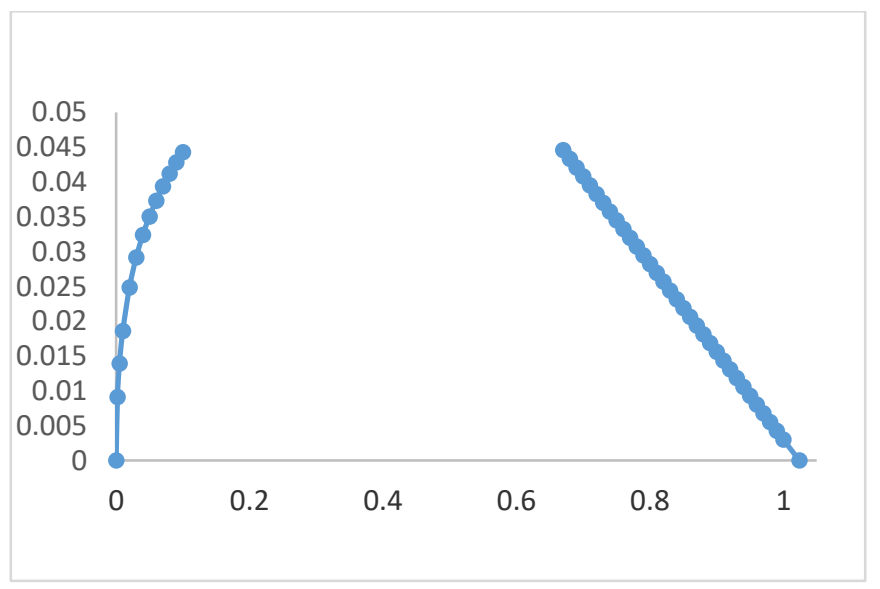

Figure 3. The fixed part of the initial airfoil.

\subsection{Local linearization method}

The Optimal airfoil from local linearization method of the previous part has been chosen. This airfoil, which was optimal airfoil at $M_{\infty}=0.76$, has been simulated over different Mach numbers.

\subsection{Elliptical airfoil}

$\mathrm{P}$ To obtain the airfoil whole shape, the fixed flat line is replaced by Elliptic shape over different $x_{c}$ (corresponding longitude of the maximum ordinate) and constant value of $y_{c}=0.06$ (maximum ordinate). The used elliptical equation is as:

$$
y=\sqrt{1-\left(\frac{x-x_{c}}{a}\right)^{2}} \times b+y_{c}
$$

Fig.4 indicates the drag coefficient at different constructed elliptical airfoils in which the middle of the airfoils is constructed by elliptic equations. The airfoil was fixed in zero angle of attack under farfield Mach number of $M_{\infty}=0.84$. As it is indicated from the results, the airfoil with elliptic central line of $x_{m}=0.425$ has the minimum value of drag force coefficient. As a result, this airfoil is the optimal airfoil of elliptical airfoil. This airfoil is simulated over different Mach numbers $M_{\infty}=$ $0.7,0.72,0.74,0.76,0.78,0.8,0.82,0.84,0.86,0.88$ and 0.9

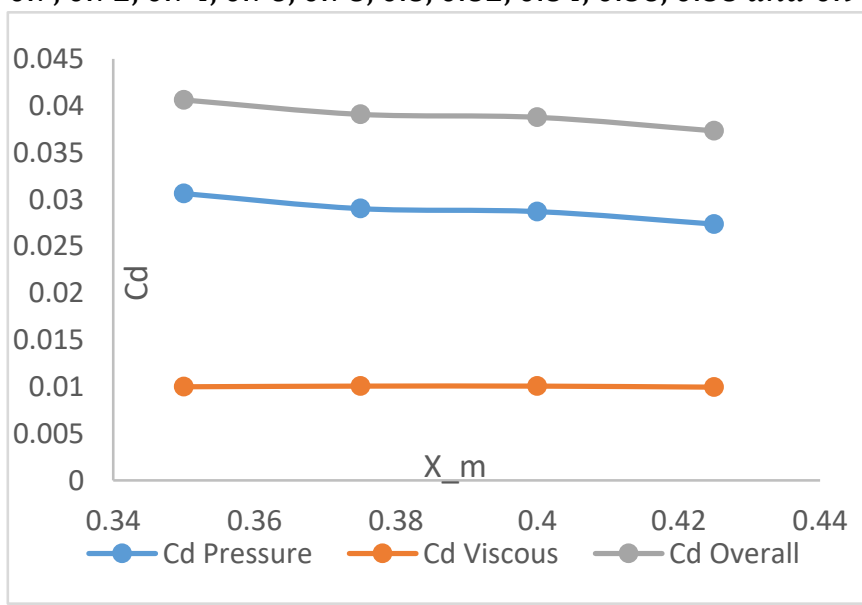

Figure 4. Drag coefficient on the constructed elliptical airfoils. 


\subsection{Airfoil Consisted from Two Inclined Lines}

This airfoil is a combined airfoil from supercritical airfoil and classic diamond shape of supersonic airfoils. The nose and tail part of the supercritical airfoil is fixed and two flat inclined lines are fixed to the maximum ordinate of the airfoil.

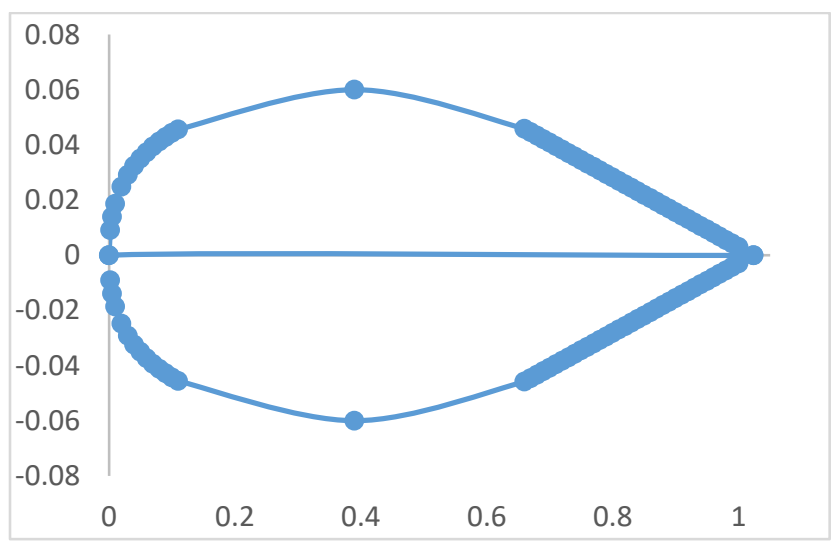

Figure 5. Geometry of the constructed airfoil.

\subsection{Comparison and Results}

Fig.6 indicates graphs of overall drag coefficient over different Mach numbers for the constructed airfoils. From the overall drag coefficient, it can be observed that the supercritical airfoil, airfoil consisted from Elliptical equation and constructed airfoil from local linearization have the same drag at low subsonic regimes but the supercritical one has a bit lower drag coefficient at high subsonic flows. However, the lowest drag coefficient at high subsonic flows belongs to the airfoil consisted from two inclined flat segments. For example, at $M_{\infty}=0.86$ the relative reduction of drag exceeds $20 \%$ and $25 \%$ rather than supercritical and optimal airfoil from local linearization airfoils, respectively. This percentage a bit reduces at the higher Mach numbers.

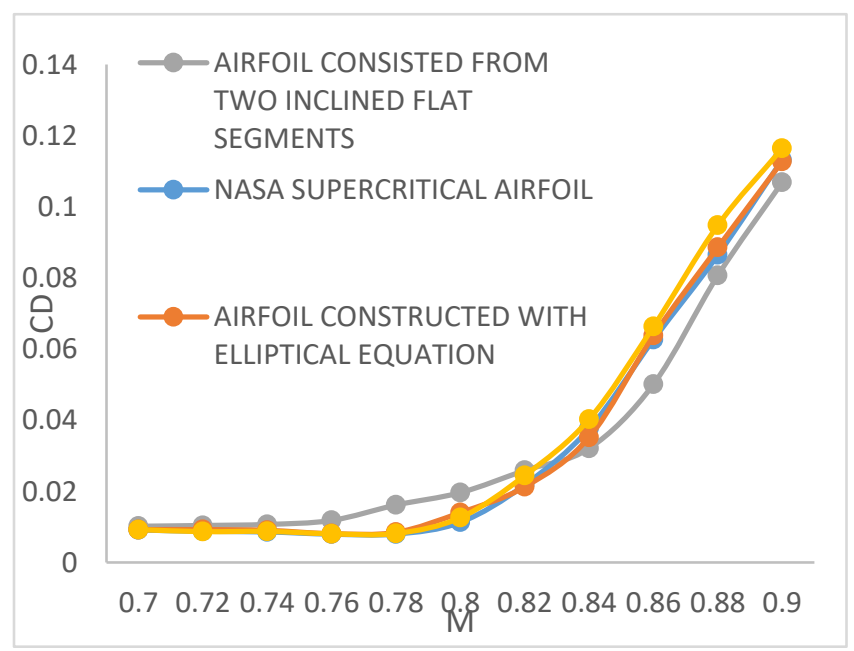

Figure 6. Drag coefficient at different Mach numbers over the constructed airfoils.

\section{Conclusion}

The first part of this research was aimed to increase $M_{C r}$. The results from an airfoil construction example shows that Local linearization provides fast convergence because of the fact that it handles many parameters. In fact, it is shown the possibility to construct the central part of the supercritical airfoil on condition of flat pressure distribution.

The second part of this research was about Drag Mitigation of Mach numbers $>M_{C r}$. The constructed airfoils were:
A) Local Linearization
B) Supercritical Airfoil
C) Elliptical Airfoil
D) Airfoil Consisted from Inclined Segments

The supercritical airfoil, Elliptical airfoil and optimal airfoil from local linearization had better performance at low Subsonic but Airfoil Consisted from Inclined Segments had the minimum Drag at high subsonic and transonic speeds. For example, at $\mathrm{M}=0.86$ the relative reduction of drag exceeds $20 \%$ and $25 \%$ rather than Supercritical and optimal airfoil of local linearization method, respectively.

\section{References}

1. R.L. Barger. and C.W. Brooks, "A streamline curvature method for design of supercritical and subcritical airfoils", NASA TN D-7770, (1974).

2. W.H. Davis, "Technique for developing design tools from the analysis methods of computational aerodynamics", AIAA Paper 79-1529, (1979).

3. M.A. Brutyan and S.V. Lyapunov, "Optimization of shape of symmetrical flat bodies with the purpose of increase of the critical Mach number", Uch. Zap. TsAGI, 12(5):10-22, (1981).

4. Sergey Takovitskii, "Aerodynamic shapes optimization on the base of method of local linearization", 7th european conference for aeronautics and space sciences (eucass), eucass 2017-95.

5. S. A. Takovitskii, "Analytical Solution in the Problem of Constructing an Airfoil with Minimum Wave Drag", Fluid Dynamics, (2003). 